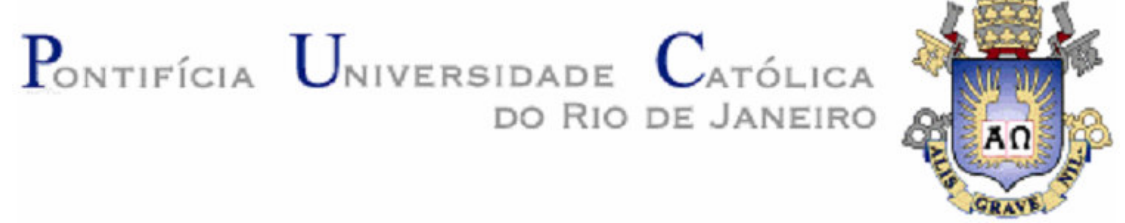

Bruno Fontes Rodrigues

\title{
Análise De Processamento Submarino na Produção De Óleo e Gás: as Novas Perspectivas sem o Uso de \\ Plataformas
}

\begin{abstract}
Dissertação de Mestrado
Dissertação apresentada ao programa de Pós-Graduação em Engenharia Mecânica da PUC-Rio como requisito parcial para obtenção do título de Mestre em Engenharia Mecânica.
\end{abstract}

Orientador: Prof. Carlos Valois Maciel Braga

Rio de Janeiro Novembro de 2011 


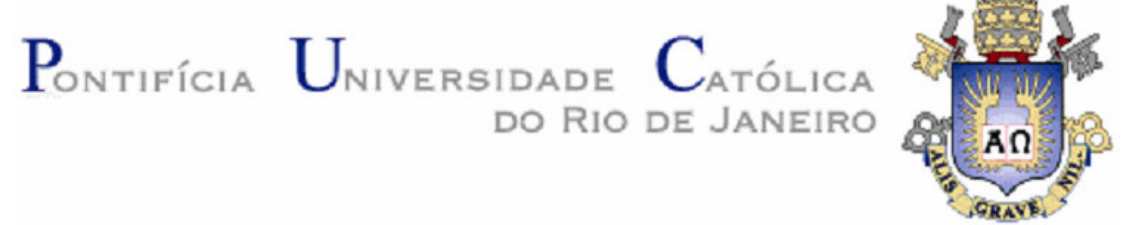

Bruno Fontes Rodrigues

\section{Análise De Processamento Submarino na Produção De Óleo e Gás: as Novas Perspectivas sem o Uso de \\ Plataformas}

Dissertação apresentada ao programa de Pós-Graduação em Engenharia Mecânica da PUC-Rio como requisito parcial para obtenção do título de Mestre em Engenharia Mecânica. Aprovado pela comissão examinadora abaixo assinada.

Prof. Carlos Valois Maciel Braga Orientador Departamento de Engenharia Mecânica da PUC-Rio

Prof. Iberê Nascentes Alves Petróleo Brasileiro S.A. - Petrobras/E\&P

Prof. Sidney Stuckenbruck Olympus Software Científico e Engenharia

Prof. Marcos Sebastião P. Gomes Departamento de Engenharia Mecânica da PUC-Rio

Prof. José Eugênio Leal Coordenador Setorial do Centro Técnico Científico PUC-Rio 
Todos os direitos reservados. É proibida a reprodução total ou parcial do trabalho sem autorização da universidade, do orientador e do autor.

\section{Bruno Fontes Rodrigues}

Graduou-se em Engenharia Mecânica na UFRJ em 2003. Concluiu Engenharia Econômica na UFRJ em 2004 e Engenharia de Petróleo na PUC-Rio em 2005. Participou de diversos congressos e hoje atua como engenheiro de dutos rígidos na empresa Technip.

Ficha Catalográfica

Rodrigues, Bruno Fontes

Análise de processamento submarino na produção de óleo e gás: as novas perspectivas sem 0 uso de plataformas / Bruno Fontes Rodrigues ; orientador: Carlos Valois Maciel Braga. $-2011$.

149 f. : il. (color.) ; $30 \mathrm{~cm}$

Dissertação (mestrado)-Pontifícia Universidade Católica do Rio de Janeiro, Departamento de Engenharia Mecânica, 2011.

Inclui bibliografia

CDD: 621 


\section{Agradecimentos}

Ao meu orientador Carlos Valois por ter aceitado o desafio deste projeto, pela confiança, estimulo, orientação e pela ajuda primordial na obtenção da bolsa de isenção.

À PUC-Rio pela concessão da bolsa de isenção, sem a qual a realização do curso não seria possível.

À funcionaria Rosely Ribeiro por toda ajuda nas situações burocráticas.

À todos os professores do curso de Mestrado de engenharia Mecânica da PUCRio que transmitiram algum conhecimento, entre eles, Carlos Valois, Marcelo Dreux, Luis Fernando Azevedo, Sidney Stuckenbruck, Fabio Braga, José Luiz Freire, Geraldo Spinelli, Arthur Braga e Rogério Espósito.

Aos professores do curso de pós graduação de Petróleo do CCE, PUC-Rio, Iberê Alves e Brenno Romano Motta Filho, pelo ensinamento, tempo disponibilizado e as dicas transmitidas.

Ao professor de pós graduação da UFRJ Elísio Caetano pelo tempo disponibilizado e ensinamentos transmitidos.

À minha esposa pelo apoio incondicional e compreensão nos momentos de dificuldade durante este período de estudo.

Aos meus pais pelo carinho e apoio sem os quais não estaria aqui.

Ao amigo Rodrigo Klim Gomes, pela companhia ao longo do curso e apoio nos momentos de desabafos e ansiedades.

À empresa Intecsea por ter permitido a execução deste mestrado durante expediente de trabalho.

À empresa Technip pela liberação do acesso ao software Pipesim sem o qual a realização deste trabalho não seria possível. 


\section{Resumo}

Rodrigues, Fontes Bruno; Braga, Carlos Valois Maciel. Análise De Processamento Submarino na Produção De Óleo e Gás: as Novas Perspectivas sem o Uso de Plataformas. Rio de Janeiro, 2011, 149p. Dissertação de Mestrado, Departamento de Engenharia Mecânica, Pontifícia Universidade Católica do Rio de Janeiro.

O petróleo tem importância inegável nos tempos atuais. Junto com outros combustíveis fósseis, representa uma considerável parcela da matriz energética da sociedade. Porém esta é uma fonte de energia não renovável. Por isso a medida que o petróleo é produzido em regiões de fácil acesso, sua fonte vai se esgotando e criando a necessidade de se explorar em regiões cada vez mais inóspitas. Diante deste cenário o processamento submarino na produção de óleo e gás tem grande relevância ao permitir a produção de petróleo em regiões onde não seria possível a produção sem esta opção. O objetivo deste trabalho é comparar 2 sistemas de processamento submarino, um com bomba multifásica e outro com separador submarino, visando identificar as oportunidades de aplicação para cada sistema e o estado da arte atual de cada tecnologia. $\mathrm{O}$ grande salto do processamento submarino será um futuro de produção sem o uso de plataformas. Cenário este que já se observa nos dias de hoje em alguns campos de gás. O campo de gás foi o primeiro a apresentar a possibilidade de produção sem o uso de plataforma por possuir energia suficiente para escoar por distâncias maiores sem necessidade de incremento artificial de pressão. Porém com o avanço da tecnologia da bomba e dos separadores submarinos, o futuro indica a aplicação deste processo também em campos de óleo. Este trabalho disponibiliza uma ferramenta simplificada para análise de escoamento multifásico de fácil acesso que permite o cálculo sem a necessidade de softwares avançados e de difícil acesso. Apesar de ser uma ferramenta simplificada é de grande utilidade para cálculos rápidos sem necessidade de detalhamento.

\section{Palavras chave}

Bomba multifásica; separador submarino; processamento óleo/gás submarino; poço à terra. 


\section{Abstract}

Rodrigues, Fontes Bruno; Braga, Carlos Valois Maciel (Advisor). Oil and Gas Subsea Processing Analysis: new Perspectives without the use of Plataforms. Rio de Janeiro, 2011, 149p. MSc Dissertation, Departamento de Engenharia Mecânica, Pontifícia Universidade Católica do Rio de Janeiro.

Oil has undeniable importance in modern times. Along with other fossil fuels, represents a considerable portion of the energy matrix of society. However this is non-renewable energy source. As the oil is been produced in areas of easy access, its source is being exhausting and creating the need to explore in inhospitable regions. Looking to this scenario subsea processing of oil and gas has great importance to allow oil production in regions where the production would not be possible without this option. The objective of this study is to compare two subsea processing systems, one with a multiphase pump and other with a separator and a monophase pump, identify opportunities of each system and the current state of the art of each technology. The leap of subsea processing will be a future of production systems without the use of platforms. This scenario already being felt today in some gas fields. The gas field was the first to present the possibility of production without the use of the platform due to the fact that gas fields has enough energy to flow over large distances without the need of artificial lifting. However, with the advancement in pump technology and subsea separators, the future indicates the application of this procedure also in oil fields. This paper provides a simplified calculation tool for multiphase flow of easy access that allows the calculation without the need of advanced commercial software. Despite being a simplified tool is useful for quick calculations.

\section{Keywords}

Multiphase pump; subsea separator; subsea oil/gas processes; subsea to shore. 


\section{Sumário}

1 Introdução .................................................................17

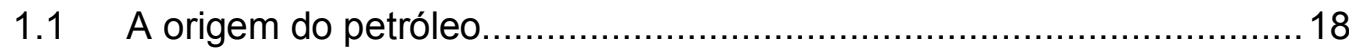

1.2 O petróleo na América do Norte ….................................................. 19

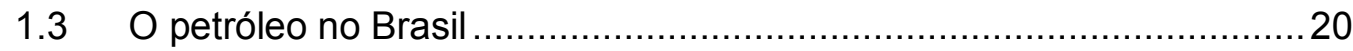

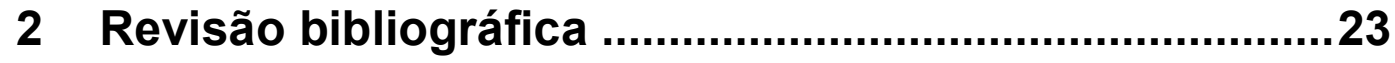

3 Bomba multifásica.........................................................25

3.1 Bomba monofásica BCS (Bomba centrífuga submersa) ...................25

3.2 Bomba multifásica ........................................................................ 26

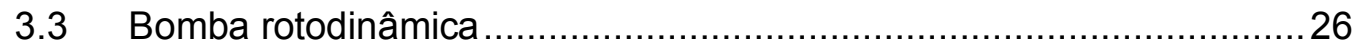

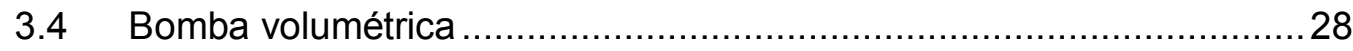

3.5 Dimensionamento de um sistema multifásico ....................................30

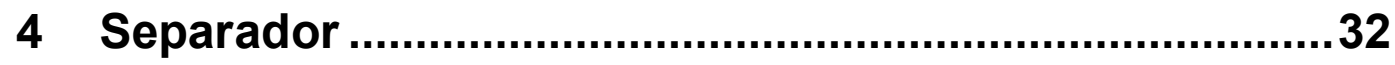

4.1 Dimensionamento de um sistema de separação................................ 36

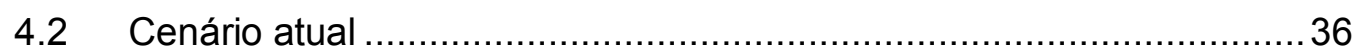

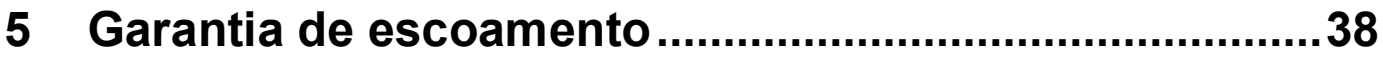

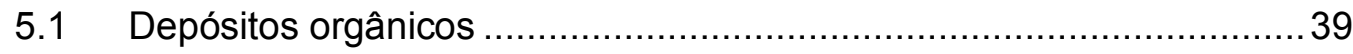

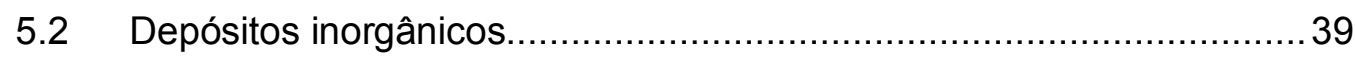

5.3 Casos e solução de garantia de escoamento ...................................40

6 Simulação .................................................................. 41

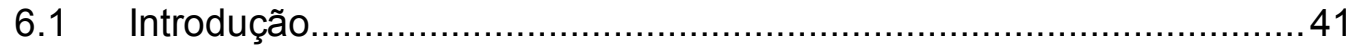

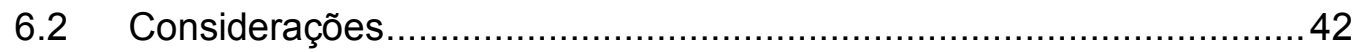

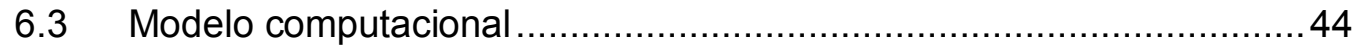

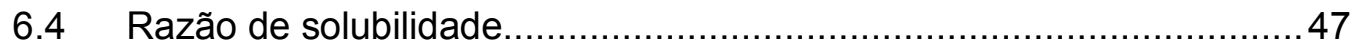

6.5 Fator volume de formação ................................................... 48

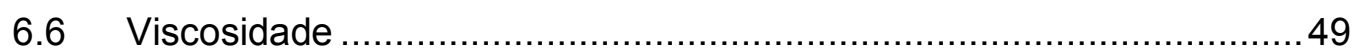

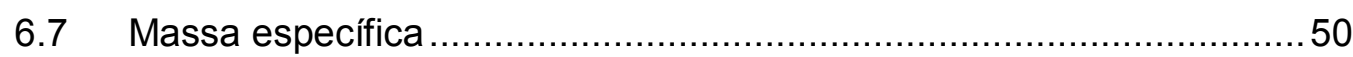

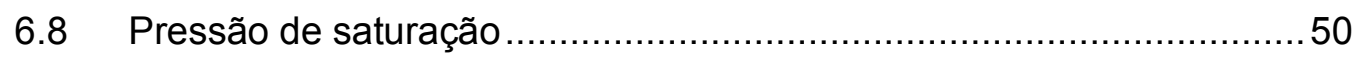

6.9 Fator de compressibilidade do gás ................................................ 51

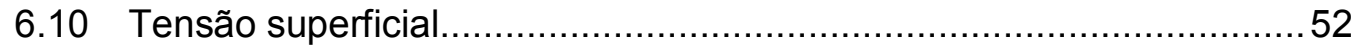

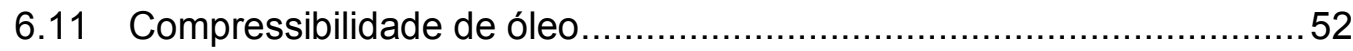

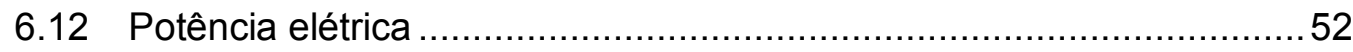

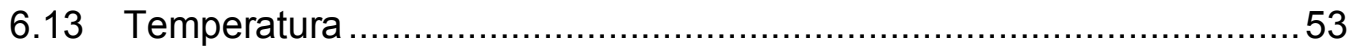

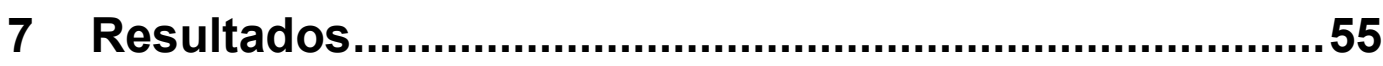

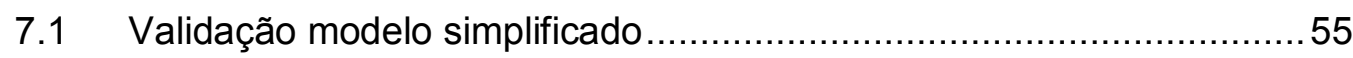




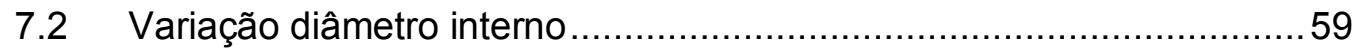

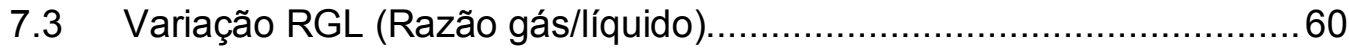

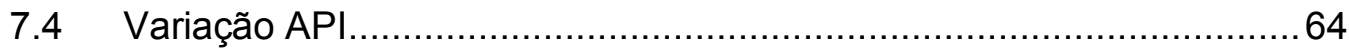

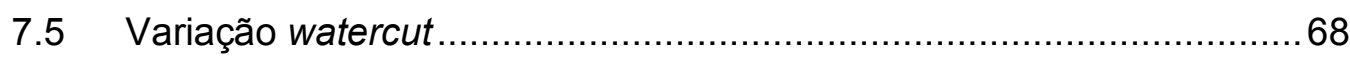

7.6 Variação densidade do gás....................................................... 72

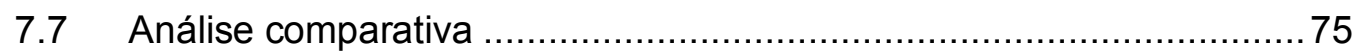

8 Conclusões e recomendações.......................................79

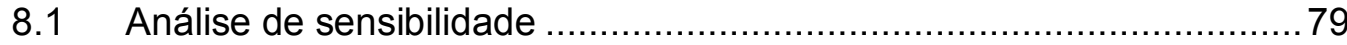

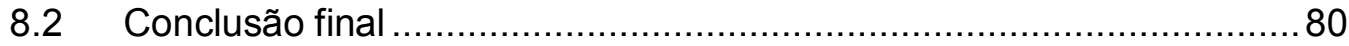

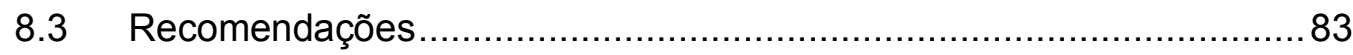

9 Referências bibliográficas ............................................ 84

Apêndice A - Escoamento Monofásico ..............................86

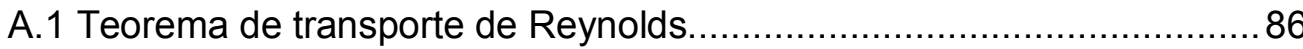

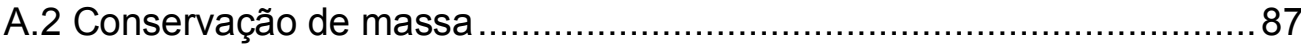

A.3 Conservação quantidade de movimento.......................................... 88

A.4 Conservação de energia........................................................... 88

Apêndice B - Introdução ao escoamento multifásico .........91

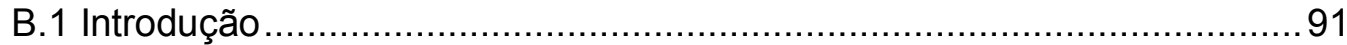

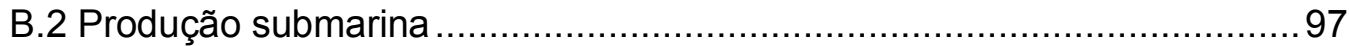

B.3 Escoamento multifásico: definições ..................................................99

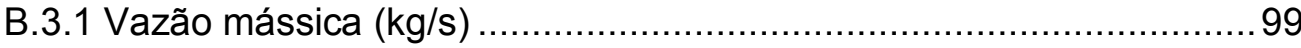

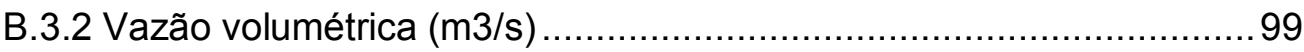

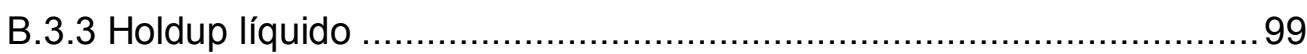

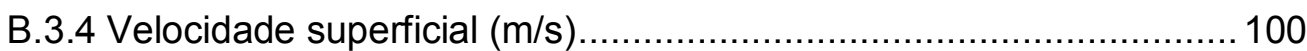

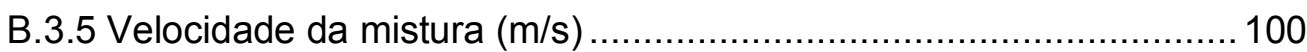

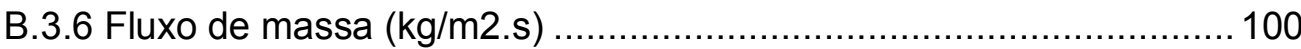

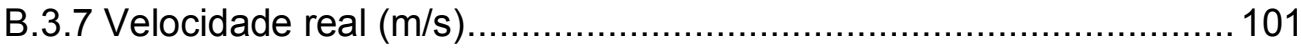

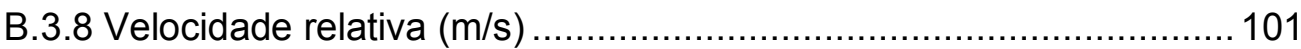

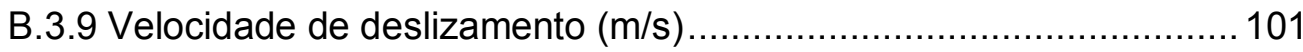

B.3.10 Concentração de massa ......................................................... 101

B.3.11 Fração de massa................................................................. 102

B.3.12 Propriedade da mistura ........................................................... 102

B.3.13 Propriedade do líquido............................................................ 102

B.3.14 Razão de solubilidade ......................................................... 102

B.3.15 Fator volume de formação ........................................................ 103

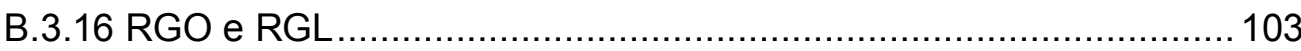


Apêndice C: Escoamento multifásico: cálculo .................105

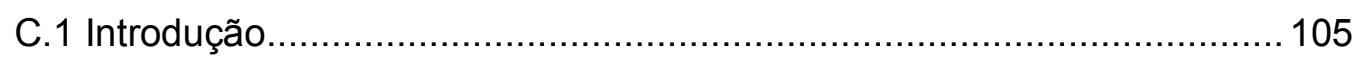

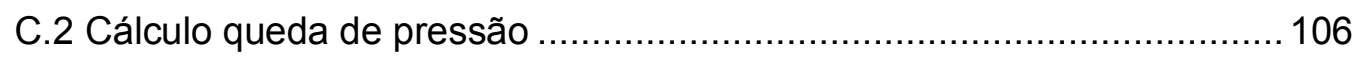

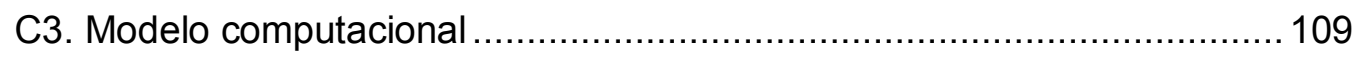

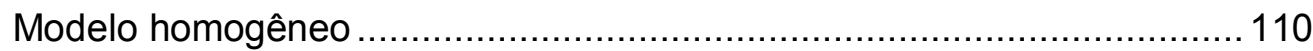

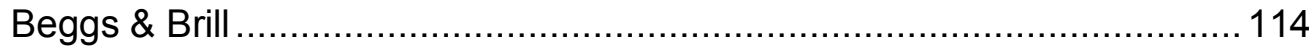

Hagedorn \& Brown ................................................................... 118

Apêndice D - Modelo escoamento multifásico ..................122 


\section{Lista de Figuras}

Figura 1-1: Início da exploração em 1901 em Summerland - California ............20

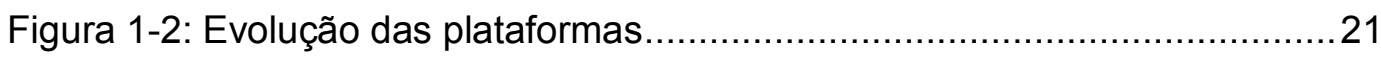

Figura 1-3: Ilustração de um campo de produção sem plataforma - Snohvit......22

Figura 3-1: Ilustração externa de uma bomba tipo hélico axial (Fabricante FRAMO)

Figura 3-2: Ilustração interna de uma bomba tipo hélico axial.

Figura 3-3: Ilustração de uma bomba tipo duplo-parafuso (Fabricante Leistritz)

Figura 4-1: llustração interna de um separador tipo VASPS (Vertical annular separation and pumping system)

Figura 4-2: Ilustração do head assembly de um separador tipo VASPS (Vertical annular separation and pumping system).

Figura 4-3: Ilustração do separador tipo Caisson (Projeto Shell e FMCParque das Conchas)

Figura 6-1: llustração do modelo utilizado no trabalho através do software PipeSim

Figura 6-2: llustração de bombas monofásicas atuais em relação a potência, vazão e eficiência

Figura 6-3: llustração de bombas multifásicas atuais em relação a potência, vazão e eficiência

Figura 7-1: Comparativos de resultado entre os modelos deste trabalho pata fim de validação do modelo MathCad

Figura 7-2: llustração da página de dados de entrada do modelo gerado em Mathcad.

Figura 7-3: Gráfico da variação da vazão de óleo x API, variando diâmetro interno do duto para bomba, separador e elevação natural

Figura 7-4: Gráfico da variação da potência requerida $\times \mathrm{API}$, variando diâmetro interno do duto para bomba, separador e elevação natural

Figura 7-5: Gráfico da variação da energia específica $x$ RGL, pressão de entrada $=150 \mathrm{bar}$

Figura 7-6: Gráfico da variação da energia específica x RGL, pressão de entrada $=75 \mathrm{bar}$

Figura 7-7: Gráfico da variação da energia específica x RGL, pressão de entrada $=25 \mathrm{bar}$ 
Figura 7-8: Gráfico da pressão de saturação e percentual de gás livre $\mathrm{x}$ RGL, pressão de entrada=150bar

Figura 7-9: Gráfico da pressão de saturação e percentual de gás livre x $\mathrm{RGL}$, pressão de entrada $=75$ bar.

Figura 7-10: Gráfico da pressão de saturação e percentual de gás livre $x$ RGL, pressão de entrada $=25 \mathrm{bar}$

Figura 7-11: Gráfico da variação da energia específica x API, pressão de entrada $=150 \mathrm{bar}$

Figura 7-12: Gráfico da variação da energia específica x API, pressão de entrada $=75 \mathrm{bar}$

Figura 7-13: Gráfico da variação da energia específica x API, pressão de entrada $=25 \mathrm{bar}$

Figura 7-14: Gráfico da variação da pressão de saturação e percentual de gás livre $x$ API, pressão de entrada=150bar

Figura 7-15: Gráfico da variação da pressão de saturação e percentual de gás livre $x$ API, pressão de entrada $=25$ bar

Figura 7-16: Gráfico da variação da energia específica x watercut, pressão de entrada $=150 \mathrm{bar}$

Figura 7-17: Gráfico da variação da energia específica x watercut, pressão de entrada $=75 \mathrm{bar}$

Figura 7-18: Gráfico da variação da energia específica x watercut, pressão de entrada $=25 \mathrm{bar}$

Figura 7-19: Gráfico da variação da pressão de saturação e percentual de gás $\mathrm{x}$ watercut, pressão de entrada $=150 \mathrm{bar}$

Figura 7-20: Gráfico da variação dad pressão de saturação e percentual de gás $\mathrm{x}$ watercut, pressão de entrada $=25 \mathrm{bar}$

Figura 7-21: Gráfico da variação da energia específica $x$ densidade do gás, pressão de entrada $=150 \mathrm{bar}$

Figura 7-22: Gráfico da variação da energia específica $x$ densidade do gás, pressão de entrada $=75 \mathrm{bar}$.

Figura 7-23: Gráfico da variação da energia específica $x$ densidade do gás, pressão de entrada $=25 \mathrm{bar}$

Figura 7-24: Gráfico da variação da pressão de saturação e percentual de gás livre $x$ densidade do gás, pressão de entrada $=150$ bar

Figura 7-25: Gráfico da variação da pressão de saturação e percentual de gás livre $x$ densidade do gás, pressão de entrada $=25 \mathrm{bar}$ 
Figura 7-26: Gráfico comparativo da região de pressão e energia específica onde bomba multifásica e separador são mais eficientes observando a

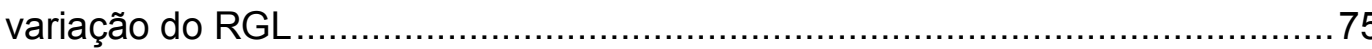

Figura 7-27: Gráfico comparativo da região de pressão e energia específica onde bomba multifásica e separador são mais eficientes observando a variação do API

Figura 7-28: Gráfico comparativo da região de pressão e energia específica onde bomba multifásica e separador são mais eficientes observando a variação do watercut.

Figura 7-29: Gráfico comparativo da região de pressão e energia específica onde bomba multifásica e separador são mais eficientes observando a

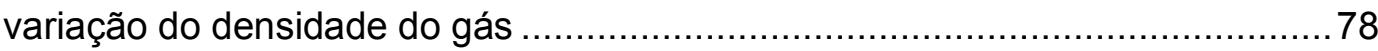

Figura B-1: Diagrama de fases típico de um hidrocarboneto..........................92

Figura B-2: Diagrama de fases para um modelo tipo black oil ..........................93

Figura B-3: Padrão de escoamento em um duto horizontal.............................95

Figura B-4: Padrão de escoamento em um duto vertical..................................95

Figura B-5: Evolução dos volumes de gás, óleo e água em função da

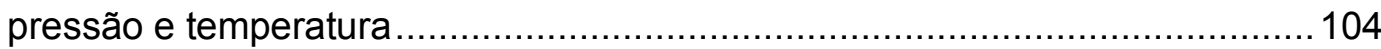

Figura C-1: Ilustração escoamento em dutos..............................................106

Figura C-2: Fator de correção do holdup líquido para a correlação de

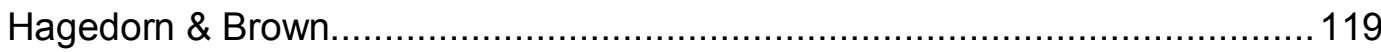

Figura C-3: Fator de correção do holdup líquido para correlação de Hagedorn \& Brown. 


\section{Lista de Tabelas}

Tabela 3-1- Dados de operação de bombas rotodinâmicas atuais....................27

Tabela 3-2-Dados de operação de bombas volumétricas atuais .........................30

Tabela 4-1-Dados de bombas monofásicdas atuais para utilização junto ao

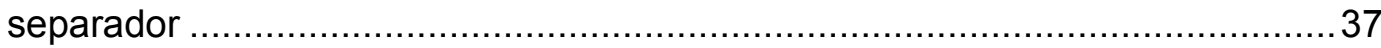

Tabela 6-1-Relação das correlações utilizadas neste trabalho...........................43

Tabela 6-2- Dados de entrada utilizados no trabalho para os parâmetros do fluido.

Tabela 6-3- Dados de entrada utilizados no trabalho para os parâmetros do duto

Tabela 6-4- Restrições do sistema consideradas neste trabalho 46

Tabela C-1- Parâmetros utilizados na correlação de Beegs \& Brill para a definição padrão de escoamento

Tabela C-2- Coeficientes utilzados na correlação de Beggs \& Brill para calculo de holdup 


\section{Nomenclatura}

\begin{tabular}{|c|c|}
\hline A & : área da seção transversal $\left(\mathrm{m}^{2}\right)$ \\
\hline a & : constante adimensional correlação Beggs \& Brill \\
\hline b & : constante adimensional correlação Beggs \& Brill \\
\hline BCS & : bomba centrífuga submersa \\
\hline $\mathrm{Bg}$ & : fator volume de formação do gás \\
\hline Bo & : fator volume de formação do óleo \\
\hline bpd & : barris por dia \\
\hline BSW & : base sedimend and water \\
\hline Bw & : fator volume de formação da água \\
\hline c & : constante adimensional correlação Beggs \& Brill \\
\hline C & : concentração, constante adimensional \\
\hline d & : dia, constante adimensional correlação Beggs \& Brill \\
\hline E & : constante adimensional correlação Beggs \& Brill \\
\hline$E$ & : energia $(\mathrm{J})$ \\
\hline EPS & : eletrical submersible pump \\
\hline $\mathrm{F}$ & : fator de atrito \\
\hline$f^{\prime}$ & : constante adimensional correlação Beggs \& Brill \\
\hline fc & : fração \\
\hline FPSO & : floating production storage and offloading \\
\hline $\mathrm{Fr}$ & : Froude \\
\hline $\mathrm{Ft}$ & : pés \\
\hline G & : gravidade $\left(\mathrm{m} / \mathrm{s}^{2}\right)$, constante adimensional correlação Beggs \& Brill \\
\hline G & : fluxo de massa $\left(\mathrm{kg} / \mathrm{m}^{2} . \mathrm{s}\right)$ \\
\hline $\mathrm{H}$ & : hora, holdup, entalpía (J) \\
\hline $\mathrm{h}$ & : coeficiente de troca de calor por convecção \\
\hline$H \& B$ & : Hagedorn and Brown \\
\hline IP & : índice de produtividade \\
\hline $\mathrm{k}$ & : condutividade térmica \\
\hline $\mathrm{Km}$ & :quilômetro \\
\hline L & : comprimento, numero admensional de Beggs \& Brill \\
\hline LDHI & : flow kinetic hydrate inhibitor \\
\hline M & : milhões \\
\hline $\mathrm{m}$ & : metro \\
\hline
\end{tabular}




\begin{tabular}{|c|c|c|}
\hline & MEG & : monoetileno glicol \\
\hline & MM & : massa molecular \\
\hline & $\mathrm{N}$ & : número adimensional \\
\hline & $P$ & : pressão (bar) \\
\hline & $q$ & : vazão $\left(m^{3} / d\right)$ \\
\hline & $Q$ & : calor $(\mathrm{J})$ \\
\hline & $\mathrm{Re}$ & : Reynold \\
\hline & RGL & : razão gás líquido $\left(\mathrm{m}^{3} / \mathrm{m}^{3}\right)$ \\
\hline & RGO & : razão gás óleo $\left(\mathrm{m}^{3} / \mathrm{m}^{3}\right)$ \\
\hline & rpm & : rotações por minuto \\
\hline & Rs & : razão de solubilidade \\
\hline & $S$ & : perímetro $(\mathrm{m})$ \\
\hline & SBMS & : sistema de bombeamento multifásico submarino \\
\hline & scf & : pés cúbicos padrão \\
\hline & $U$ & : energia interna $(\mathrm{J})$, coeficiente global de troca de calor \\
\hline & V & : volume, volts \\
\hline$\frac{\pi}{\infty}$ & v & : velocidade $(\mathrm{m} / \mathrm{s})$ \\
\hline 桀 & VASPS & : vertical annular separation and pumping system \\
\hline 莺 & W & : trabalho $(\mathrm{J})$ \\
\hline 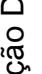 & w & : vazão mássica (kg/s) \\
\hline 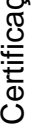 & $x$ & : fração mássica \\
\hline & & \\
\hline
\end{tabular}


Índices:

$\begin{array}{ll}\text { O } & \text { : Inclinação igual a } 0 \text { graus } \\ \text { D } & \text { : deslizamento, diâmetro } \\ \text { G } & : \text { gás } \\ \text { L } & : \text { Líquido } \\ \text { M } & : \text { mistura } \\ \text { Ns } & : \text { sem deslizamento } \\ \text { O } & : \text { óleo } \\ \text { S } & : \text { superficial } \\ \text { Tp } & : \text { duas fases } \\ \text { V } & : \text { Velocidade } \\ \text { W } & : \text { água }\end{array}$

Símbolos gregos:

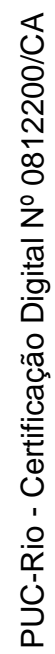

: tensão superficial entre fases

: viscosidade

: rugosidade

: ângulo

: holdup sem deslizamento

: densidade

: diâmetro

: fator de conversão 